\title{
Making Patient Engagement a Reality
}

\author{
Daphnee S. Pushparajah ${ }^{1}$
}

Published online: 24 July 2017

(c) The Author(s) 2017. This article is an open access publication

\begin{abstract}
Patients are increasingly recognised as the true customers of healthcare. By providing insights and perspectives, patients can help the wider healthcare community better understand their needs and ultimately enhance the value of healthcare solutions being developed. In the development of new medicines, for example, meaningful patient engagement can enable the pharmaceutical industry, healthcare providers and other stakeholders to achieve more meaningful health outcomes. While both the pharmaceutical industry and regulators have achieved some progress in incorporating patient perspectives into their activities, the lack of standardised best practices and metrics has made it challenging to achieve consistency and measure success in patient engagement. Practical guidance for patient engagement can facilitate better interactions between patients or patient groups and other collaborators, e.g. industry, regulators and other healthcare stakeholders. Accordingly, UCB has developed an internal model for Patient Group Engagement incorporating four key principles, based on shared ambition, transparency, accountability and respect, essential for effective collaborations.
\end{abstract}

Daphnee S. Pushparajah

Daphnee.Pushparajah@ucb.com

1 UCB Celltech, Slough, Berkshire, UK

\section{Key Points for Decision Makers}

Patient insights gained through patient engagement creates value for the healthcare ecosystem.

Mutual understanding and shared ambitions between the stakeholders are necessary for effective collaborations.

Best practices and metrics to define and measure outcomes will contribute to demonstrating the value of patient engagement.

\section{What is the Status Quo in Patient Engagement?}

Long gone are the days when the pharmaceutical industry developed medicines and related solutions on its own without collaborating with external partners, such as healthcare providers (HCPs) and academics. In particular, the industry has been recognising patients as its true customer, complementing a traditional focus on prescribers. There has been a shift towards working with patients to better understand their needs and growing awareness that scientific innovations are not always aligned with unmet patient needs. These insights have spurred greater engagement with patients across the medicines lifecycle (Fig. 1).

Working with patients creates value for the whole healthcare ecosystem. Benefits include patient insights improving the targeted product profile in discovery and research; recruitment of more of the right patients to the 
Fig. 1 Key reasons to engage with patients at specific stages of the life cycle. $H C P$ healthcare provider

$$
\begin{aligned}
& \text { - Build a patient-centred } \\
& \text { value story } \\
& \text { - Participate in regulatory } \\
& \text { approval discussions (e.g. } \\
& \text { benefit-risk proposition) }
\end{aligned}
$$

\section{- Help understand unmet patient needs and wants related to their condition \\ - Assess target product profile - treatment administration, characteristics etc. \\ - Assist in making key go-/no- go decisions \\ - Identify relevant patient populations}

Research and early development

\section{- Design patient-centred study protocols and patient- important endpoints \\ - Enhance trial recruitment, retention compliance, treatment adherence and improve patient experience during trials \\ - Learn from unsuccessful trials to improve future protocol design}

- Contribute to real-world product knowledge including benefitrisk

- Improve patient experiences in the care pathway and self- and disease management

- Support access and reimbursement

- Advise on packaging, patient (and HCP) information and education

- Guide portfolio decision making and future investment choices right clinical trials; improved patient experience during trials and ultimately the development of products that better meet patient needs. Outside of the trial environment, examples include improved disease management and effectively targeting communication and the delivery of medicines. Depending on the assumptions being validated, patient engagement is one way of getting relevant insights while appreciating there are various ways, such as large data sets and meta-analyses.

Other stakeholders in the healthcare space, such as regulators, are also engaging with patients. Since 2014, real-life experiences of patients are routinely embedded in regulatory output at the European Medicines Agency (EMA) [1]. Current patient involvement at the EMA takes the form of activities in which patients/consumers are members, alternates or observers in activities involving individual patient/consumer experts or activities requiring organisation representatives. Data show that the number of EMA events with participation from patients or patient representatives has steadily increased over the years, signalling a closer collaboration between these parties (Fig. 2).

Similarly, the US Food and Drug Administration (FDA) has stepped up engagement with patients. In 2012, the agency initiated a 5-year programme called the PatientFocused Drug Development Initiative (PFDDI), aiming to learn more from patients about the impact of their disease on their daily lives [3]. The US FDA's 'Voice of the Patient' series provides an opportunity to gather qualitative information from patients through public meetings. Both programmes focus on multiple disease areas, and, to date, psoriasis, Parkinson's disease and breast cancer, among others, have been covered.
Through these PFDDI meetings, the FDA has had the opportunity to hear directly from patients, their families and caregivers about the symptoms that matter most to them and to increase its understanding of the associated diseases. There has been valuable information on the impact of the disease on patients' daily lives, as well as their experiences with currently available treatments. Patient perspectives gained on the benefits, risks and burdens of treatments for their medical conditions can be utilised to assist the FDA in deciding how to enable drug development in specific disease areas. Underscoring the value of this interaction, the 21st Century Cures Act recently passed in the US recognises that patients should play a crucial role in the development of drugs and devices to diagnose and treat their disease [4]. In a similar vein, the FDA is considering establishing an 'Office of Patient Affairs', to be tasked with supporting and coordinating patient engagement across the agency [5].

Patient insights can indeed impact regulatory outcomes. As an illustration, the extent of psoriasis in clinical trials is measured by the Psoriasis Area and Severity Index score as a proxy for effects of the disease on quality of life. Patients, however, emphasised that the location (e.g. face, palms) had greater significance for quality of life than simply the total area covered by psoriasis. Accordingly, the EMA's guidance on psoriasis acknowledges that quality of life and the clinical severity of psoriasis often do not correlate and that, ideally, trials assessing psoriasis-specific health-related quality of life should be designed to assess the patient's perspective in the evaluation of drug effect to gauge clinical significance [6]. 


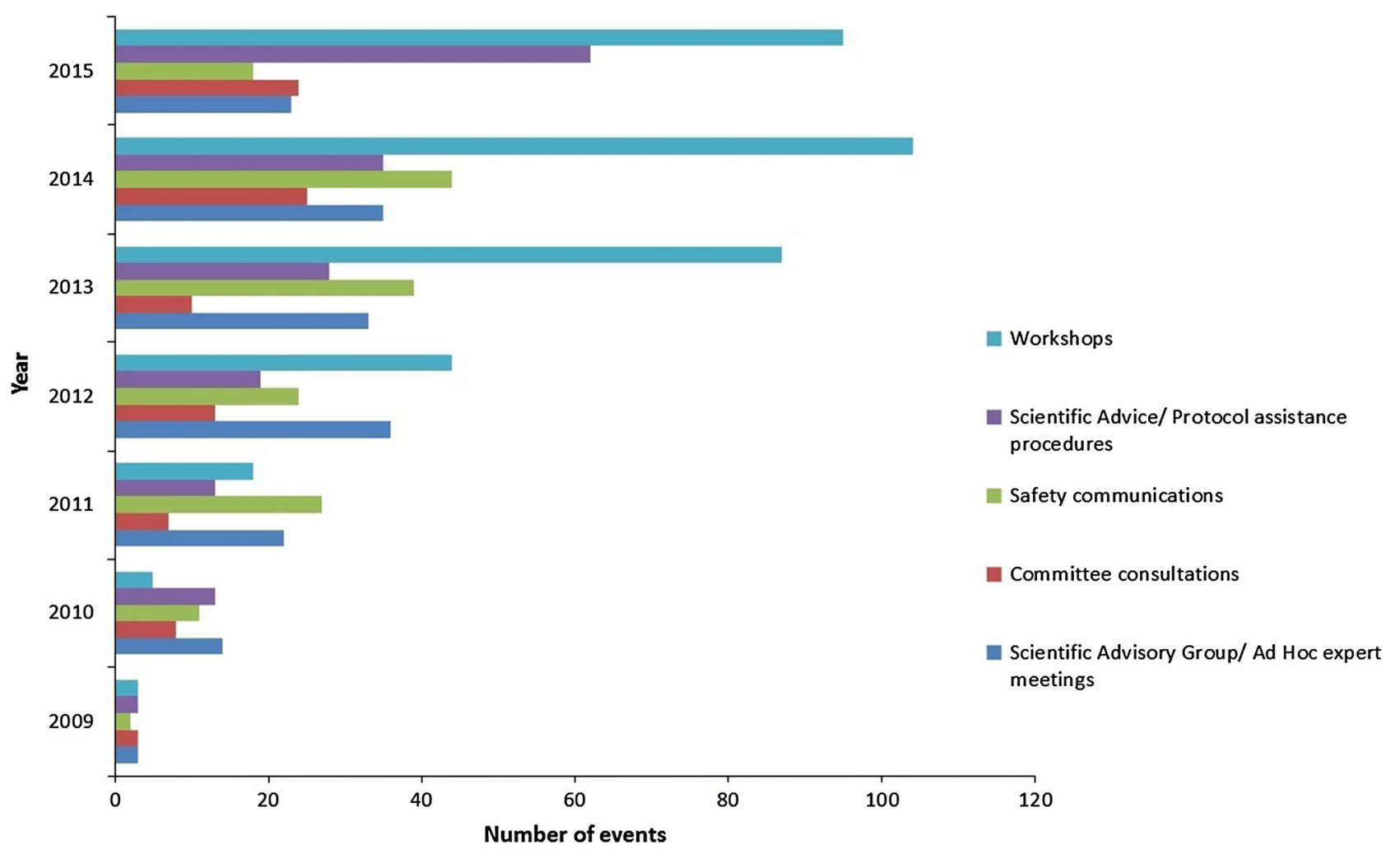

Fig. 2 Patient involvement in EMA core activities between 2009 and 2015 [2]. EMA European Medicines Agency

When the FDA approved a weight-loss treatment device for certain obese adults, the agency sponsored a survey relating to patient preferences of obesity devices. This survey revealed that a group of patients would accept risks associated with this surgically implanted device in return for the amounts of weight loss expected to be provided by the device [7]. Patient advocacy efforts from the rare disease patient community also played a crucial role in conveying the meaningful impact of a treatment for Duchenne muscular dystrophy, leading to its FDA approval [8]. These examples demonstrate how patient perspectives and preferences help the wider healthcare community to develop more effective ways to manage conditions and feed into meaningful treatment outcomes.

Other organisations incorporate direct patient engagement in their initiatives to gain first-hand understanding of patient needs and priorities. US-based FasterCures aims to integrate patient perspectives in shaping product development and influencing regulatory decisions and accelerate progress of products that patients value, from bench to bedside [9]. Similarly, the Patient-Centered Outcomes Research Institute focuses on the concept of patient-centeredness, from research to healthcare delivery, and carries out patient-centered comparative clinical effectiveness research [10].

\section{How Might Patient Engagement Evolve and What Are the Challenges?}

There are calls from different healthcare stakeholders to further enhance patient engagement in medicines development [11]. Patient involvement in clinical trials is the most cited area of collaboration between the pharmaceutical industry and patients. Contributions from patients include input into clinical protocols [12], the wording of informed consents, patient-reported outcomes tools [13], benefit-risk discussions and the development of materials to encourage patient recruitment. Beyond clinical research, areas for potential collaborations include improvement of adherence to treatments by applying patient insights to predict reasons for non-adherence.

Another area of partnership between patients and the pharmaceutical industry relates to effecting changes in public policy and advocating on topics for improving general well-being. The topics of patient empowerment, 
access to therapeutic options and policy-focused advocacy are of high interest to all stakeholders in the health environment. Prevention of cancer, as well as cardiovascular diseases (CVDs), provides examples of impactful advocacy driving changes in public policy. The combined efforts of health agencies, professional societies, patient advocates and not-for-profit organisations helped establish standards for cancer screening $[14,15]$. These have significantly improved screening test quality and resulted in reduced mortality from colorectal cancer and female breast and cervical cancer [16]. Globally, CVDs are the number one cause of death, even with the decline in mortality over the years [17, 18]. World Health Organization interventions for reducing CVDs include strategies to reduce harmful use of alcohol, comprehensive tobacco control policies, and taxation to reduce the intake of foods high in fat, sugar and salt [19]. CVD patient communities in the US and Europe have a strong presence in raising awareness of disease risk factors and advocating for policy-related changes and public campaigns [20, 21].

Often, chronic disease management and preventive health programmes focus on promoting informed lifestyle choices, risk-factor modification and active patient selfmanagement [22]. These programmes include providing patient information but, at the same time, require individuals to have a certain level of understanding and engagement. Focusing on research and development, initiatives such as the European Patients' Academy (EUPATI) aim to educate patients and encourage their involvement in the process of developing new medicines [23]. A pan-European initiative established in February 2012, EUPATI was a 5-year public-private partnership funded by the Innovative Medicines Initiative (IMI). It was patient-led, coordinated by the European Patients' Forum (EPF), with other public bodies (European AIDS Treatment Group [EATG],
Patients Network for Medical Research and Health [EGAN] and EURORDIS-Rare Diseases Europe) in key roles. The strong, multi-stakeholder consortium spanning almost 30 organisations, academia and industry has built competencies and capabilities among patient advocates and the health-interested public.

EUPATI provides objective and up-to-date information to patients and patient advocates. The programme has not only pioneered a paradigm shift towards intensified patient involvement in medicines development but has also fostered a trustful partnership between patient organisations, science and industry. Approximately 96 patient advocates have completed a training course based on a 'EUPATI Toolbox' and 'EUPATI Internet Library' in seven languages, as well as publicly available educational materials. Patient advocates trained by EUPATI now play influential roles in patient groups and collaborate with the pharmaceutical industry, regulatory authorities and health technology assessment bodies (Table 1). After becoming publicly accessible online, the EUPATI Toolbox on medicines development had more than 181,499 unique users to date. Additionally, EUAPTI has delivered four guidance documents for patient involvement with industry, ethics committees, regulators and HTA bodies. Building on its achievements, EUPATI will continue as an EPF-led multi-stakeholder partnership programme.

Patient Focused Medicines Development (PFMD) a notfor-profit, multinational coalition of patients, patient stakeholders and the pharmaceutical industry, is another initiative aiming to facilitate an integrated approach to medicines development with diverse stakeholders [24]. The PFMD ensures that the patient perspective is the starting point when identifying priorities and developing solutions to meet patients' needs. Formed as an equal collaboration among patient groups, patients and the pharmaceutical
Table 1 Comparison between roles of EUPATI-trained patients before and after the training course. Source: data on file; survey of EUPATI Fellows, December $2016(n=52)$. EUPATI European Patients' Academy

\begin{tabular}{llc}
\hline Role of patient representative & \multicolumn{2}{l}{ EUPATI training course } \\
\cline { 2 - 3 } & Before (\%) & After (\%) \\
\hline Non-active involvement as a member of patient organisation & 17 & 2 \\
Active role in a patient organisation & 62 & 71 \\
Leadership role in a patient organisation & 62 & 71 \\
Employee of a patient organisation & 25 & 23 \\
Volunteer role in a patient organisation & 60 & 67 \\
Presenting at conferences, workshops, etc. & 63 & 83 \\
Advising a pharmaceutical company & 13 & 44 \\
Advising a regulatory agency & 21 & 42 \\
Advising a reimbursement agency & 4 & 8 \\
\hline
\end{tabular}


industry, the organisation has adopted a trans-Atlantic setup that reflects its global intent. In December 2016, the IMI in Europe launched a call with many opportunities for patient engagement, including a dedicated topic on patient engagement in the medicines lifecycle [25]. The goal of this topic is to provide guidance on principles and processes for all stakeholders on the best ways to meaningfully engage patients at different stages of the medicines lifecycle.

For all these activities and some emerging guidelines [26], it should be noted that currently there is no best practice for patient engagement. Essential terminology such as patient involvement and patient engagement are often used interchangeably. However, the initiatives mentioned above should lead to greater alignment and mobilise future patient populations who are interested in gaining additional clinical/epidemiological data related to their treatments. Many pharmaceutical companies have made commitments for the responsible sharing of their clinical trial data. To this end, some pharmaceutical companies publish lay summaries of their clinical trial data on corporate websites. UCB has piloted using lay abstracts, written in plain language, in congress posters and peerreviewed publications [27-30].

Metrics on how to measure the success of patient engagement are often lacking or require greater robustness or validation. One potential example includes patient input into protocols, leading in turn to patient-friendly information that can speed recruitment to clinical trials. Nevertheless, the lack of a control group in such circumstances makes it difficult to measure the benefits of patient engagement and continues to pose challenges in obtaining budget or resources and gaining endorsement for similar activities.

The pharmaceutical industry is highly regulated and patients and other participants need to be aware of compliant and ethical rules regarding interactions with companies. As patient-focused alliances become more widespread, clarity and transparency regarding initiatives will be crucial for all contributors. Best practices for patient engagement can define the dos and don'ts of relationships. The skill set and competencies of those wishing to partner is essential in contributing to positive experiences and outcomes of any initiative. Although some highlevel industry-wide guidance is available (e.g. from the European Federation of Pharmaceutical Industries and Associations [EFPIA] and the Biotechnology Innovation Organization [31, 32]), practical tips on engagement are often lacking. UCB has developed internal recommendations, which give guidance when engaging with both patients [33] and patient groups (see box below).

\section{Case study: UCB's Patient Group Engagement (PGE) model}

\section{The Scenario}

UCB recognises that patient groups (PGs) are an essential stakeholder segment through which to enhance patient engagement throughout the entire product lifecycle. Although there were examples of positive partnerships with PGs, the overall PG engagement had scope to achieve greater clarity on objectives, with more coordinated efforts. Internally, there were challenges related to identifying the most appropriate PGs to partner with based on their interests and capabilities. Thus, guidance on PG Engagement (PGE) would enhance the organisation's capability to support strategic and impactful PG partnerships.

Moving forward, the focus was on:

- Gaining consistency and alignment in PGE

- Entering into agreements that are strategic and two-way

- Understanding that mutual benefits result in impactful value creation

- And establishing PGE as a true relationship

\section{Strategy for PGE}

The strategy focuses on increasing PGE at all stages of the lifecycle, from research through to clinical development, launch and commercialisation. The ambition is to embed these principles in all PGE activities and embark on projects that generate value for both parties. This guidance was co-created with external PG representatives and an internal cross-functional team, representing diverse viewpoints and perspectives.

Accordingly, a model was created for working with PGs that is built on four key STAR principles: (1) shared ambition (a joint vision); (2) transparency (a clear project charter); (3) accountability (well-defined roles and responsibilities); and (4) respect (longer-term continuity and responsiveness) [Table 2].

\section{Process Steps for Management of PGE}

Five 'musts' were identified for effective PGE projects (Fig. 3):

1. Define the purpose of engaging: Establish the strategic engagement objective. From this, identify when, where and which type of patient engagement is required (e.g. PGE, individual patient engagement).

2. Select the right PG partner(s): Identify PG which has shared interests and capacities to collaborate.

3. Co-create the shared project charter with the PG: Agree objectives and what success looks like for both parties, in terms of creating patient value.

4. Perform activities to plan: Allow PG enough time for engagement and manage project milestones and risks according to the project proposal.

5. Review engagement outcome: Jointly review project deliverables and experiences against the original plan with the PG. Communicate feedback and insights as appropriate. 
Table 2 STAR principles for PGE (bolded concepts are based on needs and expectations from the PG). PG Patient Group, $P G E$ Patient Group Engagement

\begin{tabular}{|c|c|c|c|}
\hline Shared ambition & Transparency & Accountability & Respect \\
\hline $\begin{array}{l}\text { Have an open and honest } \\
\text { partnership based on common } \\
\text { commitment to patient value }\end{array}$ & $\begin{array}{l}\text { Be transparent about strategy, } \\
\text { objectives and funding potential } \\
\text { with the PG }\end{array}$ & \multirow{2}{*}{$\begin{array}{l}\text { Establish a key point of } \\
\text { contact } \\
\text { Follow simplifiedand aligned } \\
\text { internal processes } \\
\text { Identify clear and effective } \\
\text { accountabilities for PGE } \\
\text { strategy ownership }\end{array}$} & $\begin{array}{l}\text { Maintain continuity of the } \\
\text { relationship beyond the } \\
\text { individual project }\end{array}$ \\
\hline $\begin{array}{l}\text { Focus on collaboration, enabling co- } \\
\text { creation }\end{array}$ & $\begin{array}{l}\text { Provide clarity to the PG about } \\
\text { UCB's internal processes, roles } \\
\text { and decision rights }\end{array}$ & & $\begin{array}{l}\text { Proactively communicate } \\
\text { and be highly responsive to } \\
\text { PG's needs }\end{array}$ \\
\hline
\end{tabular}

\section{Concluding Remarks}

In an effort to increase overall well-being, engaged stakeholders in the healthcare ecosystem, including the pharmaceutical industry, regulators and HCPs, increasingly seek to ensure that solutions developed are relevant and meaningful to patients. There is a growing number of initiatives, designed to expand the availability of educated and trained patient advocates who can partner with health stakeholders to provide valuable information in shaping the medicines lifecycle, from early research to activities after marketing authorisation and beyond.

Educated patients can play a major role in decisions related to their health and quality of life. Information and knowledge can bring about patient awareness and empowerment, leading to engagement and a more active role in decision making related to health management. Patient involvement and activation can also be utilised to establish impactful patient roles and take up leading positions (e.g. in patient groups or health advisory committees). An informed patient network can advocate and act as a catalyst to raise awareness of pertinent issues. Contributions on advocacy from trained patients might be more likely to be accepted by other 'professional' stakeholders. Importantly, the patient advocates ought to further share their knowledge, resulting in a cascade of information flow to lay patients and the broader public. A key caveat is that patients have different levels of desire to be engaged, either in their own health management or contribution to the medicines development process. There is the possible bias that a patient advocate is not representative of the entire patient population and, thus, it will be prudent to validate any insight through a variety of other means, such as database analyses or market research.

Alignment and common understanding among stakeholders on the value of patient engagement is currently lacking, although this is being addressed by several initiatives. For example, the DIA-Tufts CSDD study set out to quantify the impact of patient-centric initiatives using

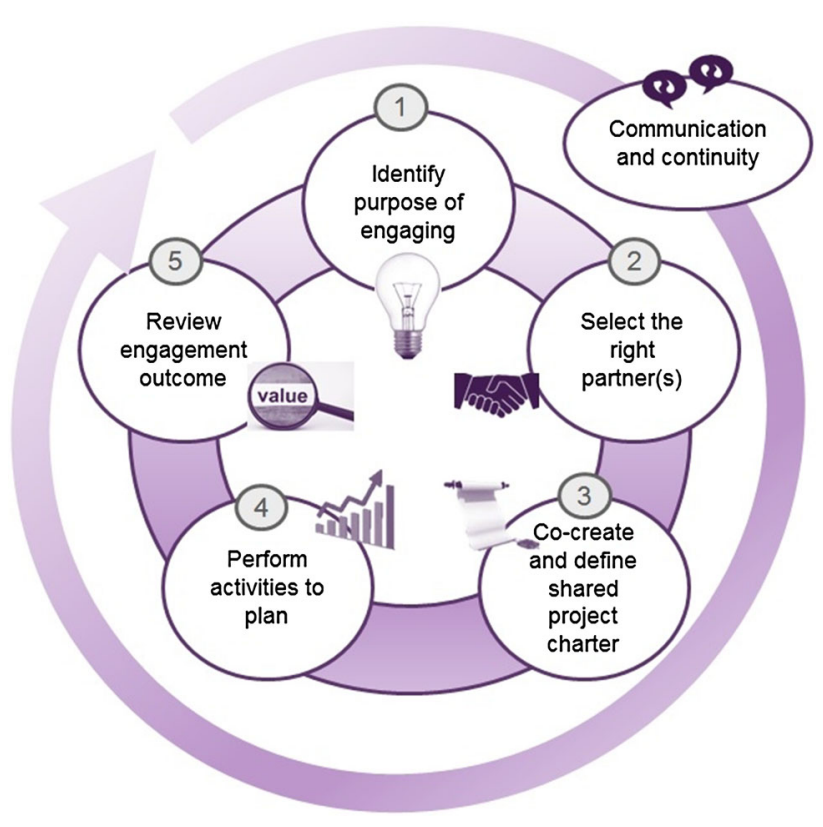

Fig. 3 Five process steps for Patient Group Engagement management. $P G$ Patient Group

return-on-engagement metrics looking at retrospective data and developing a metrics toolkit [34]. The study collected real examples of measurable benefit to drug development from patient involvement and found that metrics are not uniformly defined, making it hard to compare and generalise at this time. However, the study authors state that return-on-engagement metrics show that trial performance improves (faster planning, approval, enrolment; fewer protocol amendments); there is more positive study volunteer feedback and long-term savings across the drug development portfolio. On a related note, a valuable resource is from FasterCures, who completed an environmental scan of collaborative initiatives that have generated resources for advancing the science of patient input [35]. Frameworks, toolkits and methods related to patient engagement initiatives from over 70 organisations are listed. 
In addition to the lack of broadly accepted tools, processes and guidance on patient engagement, if and when patient engagement occurs, it is often inconsistent. Having a set of standardised best practices for engagement with patients and patient groups will go a long way toward ensuring this consistency and clarification for those who get involved. Appropriate capabilities of patients to engage and, on the other end, of those who seek patients' engagement, are both important for a successful outcome. Finally, quantitative and qualitative metrics to evaluate the impact of patient engagement practices are much needed and can in turn lead to a positive cycle of systematic and effective patient engagement. A point to consider is that sustainable partnerships are created over time, built on trust and commitment. Therefore, the results of implementing patient engagement activities (such as through UCB's PGE principles) might require time to bear fruits and outcomes.

The fundamental ethos of patient engagement is the widely quoted saying 'nothing about patients, without patients'. Thus, it makes sense for the pharmaceutical industry and other healthcare stakeholders to actively embed patient insights and perspectives in their strategic and operational plans. By seeking patient participation and meaningful involvement, the industry demonstrates respect towards the patient community, which can help build amicable sentiments between parties. Patients might no longer feel like subjects of research but as equal partners at the table, and this will surely contribute towards the ultimate goal of bettering health and advancing societal wellbeing.

Acknowledgements The author thanks all patient groups, their representatives, and UCB colleagues, especially John-Kenneth Billingsley and Lode Dewulf, who contributed towards the Patient Group Engagement principles. Thank you also to the EUPATI team and patient communities for allowing the inclusion of related data in this article.

\section{Compliance with Ethical Standards}

Conflict of interest Daphnee S. Pushparajah is an employee of UCB Pharma. The opinions expressed in this article are those of the author and do not necessarily reflect the views of her employer or organisation.

Funding The author received no financial support for the research, authorship, and/or publication of this article. Editorial assistance was funded by UCB Pharma and provided by Steve Moore, a journalist (no current affiliation).

Open Access This article is distributed under the terms of the Creative Commons Attribution-NonCommercial 4.0 International License (http://creativecommons.org/licenses/by-nc/4.0/), which permits any noncommercial use, distribution, and reproduction in any medium, provided you give appropriate credit to the original author(s) and the source, provide a link to the Creative Commons license, and indicate if changes were made.

\section{References}

1. Moulon I. Cooperation between EMA and patients' and healthcare professionals' organisations. http://www.ema.europa.eu/ docs/en_GB/document_library/Presentation/2014/07/WC500169 638.pdf. Accessed 24 May 2017.

2. Bere N. Overview of EMA's interaction with patients and consumers during 2015. http://www.ema.europa.eu/docs/en_GB/document library/Presentation/2016/02/WC500200850.pdf. Accessed 10 Feb 2017.

3. The Voice of the patient: a series of reports from FDA's patientfocused drug development initiative. http://www.fda.gov/ ForIndustry/UserFees/PrescriptionDrugUserFee/ucm368342.htm. Accessed 10 Feb 2017.

4. st Century Cures Bill Released. https://energycommerce.house. gov/news-center/press-releases/21st-century-cures-bill-released. Accessed 10 Feb 2017.

5. Enhancing patient engagement efforts across the Food and Drug Administration: establishment of a public docket; Request for comments. https://www.federalregister.gov/documents/2017/03/ 14/2017-04982/enhancing-patient-engagement-efforts-acrossthe-food-and-drug-administration-establishment-of-a. Accessed 20 Jun 2017.

6. Guideline on clinical investigation of medicinal products for the treatment of psoriatic arthritis. http://www.ema.europa.eu/docs/ en_GB/document_library/Scientific_guideline/2009/09/WC5000 03413.pdf. Accessed 10 Feb 2017.

7. Survey on patient risk tolerance. http://www.fda.gov/downloads/ AdvisoryCommittees/CommitteesMeetingMaterials/MedicalDevi ces/MedicalDevicesAdvisoryCommittee/Gastroenterology-Uro logyDevicesPanel/UCM302781.pdf. Accessed 10 Feb 2017.

8. Mukherjee S. The FDA just made its most controversial drug approval of the year. http://fortune.com/2016/09/19/fda-drugapproval-exondys-51/. Accessed 10 Feb 2017.

9. About FasterCures. http://www.fastercures.org/about/fastercures/. Accessed 10 Feb 2017.

10. PCORI: About Us. http://www.pcori.org/about-us. Accessed 10 Feb 2017.

11. Hoos A, Anderson J, Boutin M, Dewulf L, Geissler J, Johnston G, et al. Partnering with patients in the development and lifecycle of medicines: a call for action. Ther Innov Regul Sci. 2015;49(6):929-39.

12. Holm KE, Casaburi R, Cerreta S, Gussin HA, Husbands J, Porszasz J, et al. Patient involvement in the design of a patientcentered clinical trial to promote adherence to supplemental oxygen therapy in COPD. Patient. 2016;9(3):271-9.

13. de Wit M, Kirwan JR, Tugwell P, Beaton D, Boers M, Brooks P, et al. Successful stepwise development of patient research partnership: 14 years' experience of actions and consequences in Outcome Measures in Rheumatology (OMERACT). Patient. 2017; 10(2):141-52.

14. CDC. Breast cancer. Atlanta, GA: US Department of Health and Human Services, CDC; 2011. http://www.cdc.gov/cancer/breast. Accessed 10 Feb 2017.

15. Seeff L, Nadel M, Blackman D, Pollack LA. CDC. Colorectal cancer test use among persons aged $\geq 50$ years-United States, 2001. MMWR 2003;52:193-6. https://www.cdc.gov/mmwr/ preview/mmwrhtml/mm5210a2.htm. Accessed 10 Feb 2017.

16. CDC. The guide to community preventive services. Atlanta, GA: US Department of Health and Human Services, CDC; 2011. MMWR Morb Mortal Wkly Rep. 2011;60(19);619-623.

17. Koppaka R. Ten great public health achievements-United States, 2001-2010. https://www.cdc.gov/mmwr/preview/ mmwrhtml/mm6019a5.htm. Accessed 10 Feb 2017.

18. European Cardiovascular Disease Statistics. https://www. escardio.org/The-ESC/What-we-do/Initiatives/EuroHeart/2012- 
European-Cardiovascular-Disease-Statistics\#. Accessed $10 \mathrm{Feb}$ 2017.

19. Cardiovascular diseases (CVDs): Fact sheet. http://www.who.int/ mediacentre/factsheets/fs317/en/. Accessed 10 Feb 2017.

20. A public health action plan to prevent heart disease and stroke. https://www.cdc.gov/dhdsp/action_plan/pdfs/action_plan_full.pdf. Accessed 10 Feb 2017.

21. Public policy approaches to the prevention of heart disease and stroke. http://circ.ahajournals.org/content/124/23/2560. Accessed 10 Feb 2017.

22. Adams RJ. Improving health outcomes with better patient understanding and education. Risk Manag Healthc Policy. 2010;3:61-72.

23. What is EUPATI? https://www.eupati.eu/what-is-eupati/. Accessed 10 Feb 2017

24. About PFMD. http://patientfocusedmedicine.org/about-pfmd/. Accessed 10 Feb 2017.

25. Patient perspectives in medicines lifecycle. https://ec.europa.eu/ research/participants/portal/desktop/en/opportunities/h2020/topics/ imi2-2016-10-07.html. Accessed 10 Feb 2017.

26. Kirwan JR, de Wit M, Frank L, Haywood KL, Salek S, BraceMcDonnell S, et al. Emerging guidelines for patient engagement in research. Value Health. 2017;20(3):481-6.

27. Schiff M, Aris M, Saunderson S, Mountian I, Hartley P. SAT0631-HPR chronic disease and self-injection: ethnographic investigations into the patient experience during treatment. Ann Rheum Dis. 2016;75(Suppl 2):1291-2.

28. Gossec L, Saraux A, Chauvin P, Poussière M, de Chalus T, Saulot $\mathrm{V}$, et al. THU0619 lifestyle beliefs of 672 patients with rheumatoid arthritis or axial spondyloarthritis. Ann Rheum Dis. 2016;75(Suppl 2):417.

29. Gossec L, Chauvin P, Hudry C, Poussière M, de Chalus T, Saulot $\mathrm{V}$, et al. thu0620 gender, disease activity, anxiety and depression levels are related to the levels of fears of patients with rheumatoid arthritis or axial spondyloarthritis: a cross-sectional study of 672 patients. Ann Rheum Dis. 2016;75(Suppl 2):417-8.

30. Pushparajah DS, Manning E, Michels E, Arnaudeau-Bégard C. Value of developing plain language summaries of scientific and clinical articles: a survey of patients and physicians. UCB, data on file.

31. EFPIA code of practice on relationships between the pharmaceutical industry and patient organisations. http://transparency. efpia.eu/uploads/Modules/Documents/code_po2011.pdf. Accessed 10 Feb 2017.

32. BIO guiding principles for interaction with patient advocacy organizations. https://www.bio.org/bio_patient_engagement_ principles. Accessed 10 Feb 2017.

33. Dewulf L. Patient engagement by pharma-why and how? A framework for compliant patient engagement. Ther Innov Regul Sci. 2015;49(1):9-16.

34. Capturing the value of patient engagement. http://www.diaglobal. org/en/resources/tools-and-downloads/dia-tufts-csdd-study. Accessed 20 Jun 2017.

35. Science of patient input resources. http://www.fastercures.org/ programs/patients-count/science-of-patient-input-resources/. Accessed 20 Jun 2017. 